\title{
Correction: Estimation of the hydraulic parameters of leaky aquifers based on pumping tests and coupled simulation/optimization: verification using a layered aquifer in Tianjin, China
}

\author{
Gang Zheng ${ }^{1,2} \cdot$ Da Ha $^{1,2,3} \cdot$ Hugo Loaiciga ${ }^{3} \cdot$ Haizuo Zhou $^{1,2} \cdot$ Chaofeng Zeng ${ }^{4} \cdot$ Huihui Zhang ${ }^{3,5}$ \\ Published online: 15 November 2019 \\ (C) Springer-Verlag GmbH Germany, part of Springer Nature 2019
}

Correction: Hydrogeology Journal https://doi.org/10.1007/s10040-019-02021-z

The correct email address for the corresponding author (Haizuo Zhou) is: hzzhou@tju.edu.cn

The online version of the original article can be found at https://doi.org/ 10.1007/s10040-019-02021-Z

Haizuo Zhou

hzzhou@tju.edu.cn

1 School of Civil Engineering, Tianjin University, Tianjin 300072 , China

2 Key Laboratory of Coast Civil Structure Safety of Ministry of Education, Tianjin University, Tianjin 300072, China

3 Department of Geography, University of California, Santa Barbara, CA 93106, USA

4 Hunan Provincial Key Laboratory of Geotechnical Engineering for Stability Control and Health Monitoring, Hunan University of Science and Technology, Xiangtan 411201, Hunan, China

5 School of Resources and Environmental Sciences, Wuhan University, Wuhan 430079, China 